山्山FFRANÇAISE

$\supset \mathrm{DE}$

$\stackrel{1=1}{\simeq}$ PÉDAGOGIE

\section{Revue française de pédagogie}

Recherches en éducation

154 | janvier-mars 2006

La construction des politiques d'éducation : de nouveaux rapports entre science et politique

\title{
De nouveaux rapports entre science et politique : le cas des programmes scolaires
}

New relations between science and politics: school curricula

Neue Verhältnisse zwischen Wissenschaft und Politik: der Fall der Lehrpläne

Nuevas relaciones entre ciencia y política : el caso de los programas escolares

Dominique Raulin

\section{(2) OpenEdition \\ Journals}

Édition électronique

URL : http://journals.openedition.org/rfp/127

DOI : $10.4000 /$ rfp. 127

ISSN : 2105-2913

Éditeur

ENS Éditions

Édition imprimée

Date de publication : 1 mars 2006

Pagination : $61-72$

ISBN : 2-7342-1039

ISSN : 0556-7807

Référence électronique

Dominique Raulin, «De nouveaux rapports entre science et politique : le cas des programmes

scolaires », Revue française de pédagogie [En ligne], 154 I janvier-mars 2006, mis en ligne le 01 mars

2010, consulté le 30 avril 2019. URL : http://journals.openedition.org/rfp/127 ; DOI : 10.4000/rfp.127 


\title{
De nouve a UX rapports entre science et politique: le c aS deS program meS Scolaires
}

\author{
Dom inique Raulin
}

Les programmes scolaires ne sont pas Considérés Comme des objets soumis aux alternances politiques: on aurait tendance à Croire qu'ils sont a-poltiques et qu'ils sont le domaine réservé des experts. Depuis une vingtaine d'années, la prise en Compte par l'ÉCole d'attentes de la société, de plus en plus nombreuses et variées, a imposé aux responsables politiques de faire des Choix, les amenant parfois à négliger les références scientifiques. La rédaction du socle Commun de Connaissances et de Compétences devrait obliger à trouver un nouvel équilibre.

Descripte urs (TEE): Conseil national des programmes, développement de l'éducation, France, programme d'enseignement, $1990-1999$.

$\mathrm{D}$ ans la deuxième partie $d u \quad x x^{e}$ siècle, beaucoup de $m$ inistres de l'éduc aion ont fait des réformes, se préoc opant surtout d'adapter et de moderniser le fonctionnement des établissements et des enseignements. Après l'allongement de la scolarité obligatoire (1959) et la Création des b ac cataréats technologiques (1967), la première grande réforme est la Création du Collège unique, institué en 1975 : aıx termes de la loi Haby, la formation de la première partie de l'enseignement secondaire est unifiée. Au début des années 1980 , le Collège est encore au Centre des préoccupations : Louis Legrand remet à Alain Savary, son rapport Pour un Collège démocratique dont les recomm andations tendent à renforcer le Collège unique (Legrand, 19 \&). En 198 , pour répondre à la demande économique de diplôm és de niveau IV (baccalauréat) et à la demande sociale d'augmentation du niveau général de formation, les baccalauréats professionnels sont Créés (1). C'est aussi à Cette époque que Jean-Pierre Chevènement, alors ministre de l'Éducation nationale, lance le slogan de $80 \%$ d'une tranche d'âge au niveau du baccalauréat. Au début des années $19 \boxplus$, Lionel Jospin entame la rénovation pédagogique des lycées dans la logique de la loi d'orientation du 11 juillet 1989 uqi plaçait l'élève au centre du système éducatif. La préoccupation est d'accueillir et de gérer au mieux le nouveau flux d'élèves qui atteignent le lycée puis l'enseignement supérieur (2).

Alors que l'éCole a été longtemps avec la famille le lieu privilégié où se forgeait l'éducation des jeunes, 
le développement des médias en tous genres bouleverse Cette situation, donnant un ac Os beaucoup plus diversifié aux Connaissances: les réformes de structures n'ont pas pris en Compte Cette nouvelle réalité. Parallèlement, la masse des savoirs et des Créations connaît depuis la fin $d u \quad X X^{e}$ siècle un ac coissement sans précédent.

L'école à donc à faire face à la concurrence extérieure d'autres sources de savoirs et à la difficulté du Choix de Ce qui doit être enseigné. Malgré Cette réalité, les responsables administratifs ou politiques de l'éduc aion ont Continué pendant longtemps à se désintéresser de la question des Contenus : les programmes d'enseignement étaient l'objet d'un ac ord tacite. La rupture peut être située au milieu des années 1970 quand le premier choc pétrolier s'est traduit par des vagues de licenciement et par une augmentation très importante du nombre de chômeurs. On s'est alors volontiers tourné vers l'École pour lui reprocher son Conservatisme, son inaptitude aux Changements, etc., l'inadéquation de ses diplômes aux exigences nouvelles des entreprises, etc. Ce dernier reproche est d'autant plus injuste que les diplômes professionnels sont définis depuis les années 1950 dans le cadre des Commissions professionnelles Consultatives (CPC) (3) dans lesquelles sont représentés les employeurs, les salariés, les pouvoirs publics, etc.

\section{RAPPEL HISTORIQUE}

À partir de là, la modernisation de l'école va devenir une préoccupation majeure des différents gouvernements qui se succèdent.

En 1985 le sociologue Pierre Bourdieu remet au Président de la République le rapport Propositions pour l'enseignement de l'avenir, au nom du Collège de France. On peut y lire : "L'inertie struCturale du système d'enseignement qui se traduit par un retard plus ou moins grand selon les moments et selon les domaines, des contenus enseignés par rapport aux acquis de la recherche et aux demandes de la société, devrait être méthodiquement Corrigée par des interventions réglementaires ou des incitations indirectes visant à favoriser la révision des programmes, des manuels, des méthodes et instruments pédagogiques" (Collège de France, 1985 p. 31). Le cadre est donné : les programmes scoIaires ne peuvent continuer à faire abstraction des développements de la science et de l'évolution de la société.
À partir de là, l'enjeu va Consister à Chercher un équilibre entre des options Contradictoires soutenues par des universitaires, des intellectuels et des responsables politiques.

À partir de 1988 Lionel Jospin, ministre de l'Éducation nationale, s'ins Crivant dans Ia logique du rapport du Collège de France, ouvre le Chantier de la rénovation des programmes d'enseignement. Dans Chaque discipline, il confie à un universitaire de renom, la présidence d'une mission de réflexion qui " $s$ 'étend de l'enseignement élémentaire à l'université " (4) Sous la Co-présidence de François Gros (physicien) et de Pierre Bourdieu, Ces onze présidents forment la "Commission Bourdieu-Gros". Dans un premier temps, Celle-Ci va rédiger et rendre public un texte d'orientation Principes pour une réflexion sur les Contenus d'enseignement (1989): il propose sept principes et suggère la création du Conseil national des programmes d'enseignement qui "aura pourtâche de mettre en œuvre l'ensemble des [sept] principes". La plupart d'entre eux sont aujourd'hui rentrés dans des habitudes des rédacteurs de programmes: l'actualisation des programmes, la hiérarchisation des savoirs, la diversification des approches, les enseignements donnés en Commun, etc. (5) Dans Chaque discipline, le président, assisté d'un groupe qui comporte des personnalités d'origines très diverses, reconnues pour leurs Compétences, rédige un rapport qui est remis au ministre au Cours du deuxième trimestre 1989 .l présente un état des lieux de l'enseignement de la discipline et des propositions de modifications qui sont fondés sur une Consultation très large des enseignants: Ceux-Ci ont été invités à répondre à un questionnaire individuel et des synthèses ont été faites par les inspecteurs territoriaux au niveau de chaque académie.

Enfin, la loi d'orientation du 10 juillet 1989 Crée le Conseil national des programmes (CNP) : c'est l'aboutissement de Cette phase de réflexion sur les méthodes d'élaboration des programmes: dans son principe, le CNP doit permettre que soient mieux pris en Compte dans les programmes, les évolutions scientifiques et les attentes de la société.

En quelques années (entre 1985 et 1990 - le CNP est Créé par un décret du 23 février 1990), le Contexte d'élaboration des Contenus d'enseignement a donc totalement Changé : d'une situation de monopole où des groupes restreints réunis autour d'ins. pecteurs généraux, rédigeaient les programmes, on entre au début des années 1990 dans une période 
où une instance extérieure va jouer un rôle de réguIation transversale. Les responsables politiques espèrent ainsi améliorer la qualité scientifique des programmes et mieux répondre aux attentes renouvelées de la société. Ils n'ont sans doute pas perçu à l'époque les Conséquences de la Création du CNP au lieu de recourir à une instance - l'Inspection générale de l'Éducation nationale (IGEN) - dont la position dans la hiérarchie de l'Éducation nationale est Connue de tous, pour écrire les programmes, on met en place une instance "experte " transversale dont ni le positionnement hiérarchique, ni la mission ne sont très précis: "Le CNP donne des avis et adresse des propositions au ministre de l'éducation nationale sur la Conception générale des enseignements, les grands objectifs à atteindre, l'adéquation des programmes et des champs disciplinaires, à Ces objectifs et leur adaptation au développement des Connaissances " (France, 1990, article 2). Ces impréCisions ne vont pas manquer d'avoir des effets sur le fonctionnement et l'efficacité du CNP.

\section{LE CONSEIL NATIONAL DES PROGRAMMES (CNP)}

\section{Une in stance transve rsale}

Aux termes de son décret de Création, le CNP com porte vingt-deux membres, Choisis pour leurs Com pétences. Leur m andat est de Cinq ans, renouvelable une fois. Depuis 1990 , différents équilibres ont toujours été respectés dans le choix des membres: entre les disciplines, au besoin en faisant appel à des Chargés de mission Complémentaires, entre les ordres d'enseignement (primaire, secondaire, supérieur), entre les fonctions (enseignants, inspecteurs); enfin, sont également présentes des personnalités qui ne sont pas issues de l'éducation (Chercheurs, acteurs de la société Civile, etc.). En 2004, le CNP ( $m$ embres et Chargés de mission) Comportait trois inspecteurs généraux, une directrice d'école, trois enseignants du second degré (mathématiques, français, économie-gestion), trois enseignants en Institut universitaire de form ation des maîtres (IUFM), un professeur des Classes préparatoires aux grandes écoles (CPGE), un éCrivain-essayiste, un représentant de la soCiété Civile et dix enseignants-CherCheurs; ils se répartissent entre les disciplines de la façon suivante : deux pour le français, un pour les arts, quatre pour la philosophie, trois pour les mathématiques, deux pour la physique-Chimie, deux pour l'histoiregéographie, trois pour l'économie et le droit, un pour les langues vivantes, un pour la technologie, deux pour les sciences de la vie et de la terre (6).
Selon les avis rendus par le Conseil d'État suite à des recours déposés sur la validité de Certains programmes, le Conseil national des programmes est un organisme Consultatif ${ }^{7}$ dont le fonctionnement est parfaitement Codifié : délai d'envoi des Convocations, précision de l'ordre du jour, quorum, etc.

Avec la nomination de LuC Ferry (Cf. infra), le CNP va pleinement jouer son rôle de régulation en émettant des avis Collégiaux sur les projets de programmes qui lui sont présentés. Les avis sont publics et Communiqués aux membres du Conseil supérieur de l'éducation (Cf. infra).

\section{Le risque de l'opposition entre eXperts}

Les projets de programmes sont rédigés sous la responsabilité d'universitaires avec l'aide de groupes qu'ils Constituent, initialement Groupes techniques disciplinaires (GTD), transform és depuis en Groupes d'experts pour les programmes scolaires (GEPS). Ils sont Constitués d'une douzaine de personnes qualifiées: enseignants, inspecteurs, universitaires. Tout comme leur président, les membres des groupes ont la Conviction de faire des choix justes: ils Comprennent et acceptent $m$ al les remarques que le CNP peut formuler sur Ceux-Ci. En différentes occasions, se sont développés des désaccords entre les groupes d'experts d'une part et l'instance experte - le CNP - d'autre part.

L'exemple le plus marquant est Celui des programmes de philosophie qui ont Connu de nombreuses réformes avortées entre 1992 et 2003. Dans son avis sur les programmes des séries générales (L, ES et S) du $28 \mathrm{~m}$ ai 2002 , le CNP fais ait l'analyse suivante : "Au Cours des quarante dernières années, les programmes de philosophie ont évolué, lentement $m$ ais sûrement, dans le sens de l'indéterm ination [...] Depuis longtemps, le CNP, pour remédier à Cette situation préjudiciable aux élèves comme à la $\mathrm{dis}$ Cipline, réclam ait que les groupes d'experts Chargés de rédiger les programmes de philosophie fassent l'effort de déterminer davantage Ces programmes en vue de mieux réaliser deux objectifs : transmettre un minimum de Culture commune aux élèves, notamment en matière d'histoire de la philosophie; rendre l'évaluation au baccalauréat moins aléatoire". Le projet Concerné par Cet avis n'a pas abouti et un nouveau groupe d'experts a été mandaté.

Dans son avis du 22 avril 2003 sur le nouveau projet, le CNP indique : "Le CNP estime que Ce texte ne représente pas encore une avancée suffisante 
dans le domaine de la détermination et ne protège donc pas les enseignants et leurs élèves contre les risques d'une évaluation aléatoire $a u b$ ac cataréat. Mais les rédactions suc ossives des programmes de philosophie ont suscité de tels différends qu'il n'apparaît plus possible de faire évoluer cet enseignement par la voie des programmes ". L'opposition reste entière entre le CNP et une grande part de la Corporation Constituée d'universitaires, de professeurs de lyCées et d'inspecteurs: elle porte à la fois sur la Conception de la discipline et sur Celle d'un programme d'enseignement scolaire.

Au moment de l'écriture des programmes de Collège entre 1995 et 1998 , une situation analogue s'est révélée à propos de la technologie. En 1996, l'avis du CNP sur le programme du CyCle Central (Classes de $5^{\mathrm{e}}$ et de $4^{\mathrm{e}}$ ) $\mathrm{m}$ arque son désaccord dans les termes suivants : "le CNP s'interroge sur plusieurs points : le caractère très "enseignement professionnel "de Ce programme [...]; Ia Cohérence globale et la progression des scénarios de projets et des unités informatiques [...]; la différence de présentation entre les unités informatiques et les scénarios de projet, avec pour ces derniers une absence de toute "notion" précise à acquérir. II serait pour le moins paradoxal que la technologie devienne la seule discipline où n'apparaisse aucune exigence de Connaissance [...]; Ia quasi-absence Concomitante de fléchage des relations avec les autres disciplines [...]; l'écart "surprenant» entre les compétences instrumentales visées en fin de cycle qui relèvent pour l'essentiel du "bricolage " [...] et la référence permanente aux pratiques en entreprises; le volume trop important des compétences visées". Comme pour la philosophie, la corporation enseignante au sens large est plutôt favorable au projet du groupe technique. Sur la base de Cet avis du CNP dont il faut rappeler qu'il est public, un accord a pu être trouvé sans que soit tranchée la question des références qui servent à définir les Contenus de Cette discipline : d'un Côté, le GTD, fidèle à l'histoire de la technologie, se réfère aux pratiques de l'entreprise ; de l'autre, le CNP souhaite l'orienter plus nettement vers la technologie industrielle et particulièrement la réalisation, reprenant à son compte les propos de Lucien Gém in ard en 1977 : "Le but de la fabrication n'est pas l'objet lui-même, m ais ce que la fabrication de l'objet aura eu comme effet sur divers grands aspects du développement de l'enfant [...] Le développement étant recherché par l'action, par l'acte et par la parole" (Cité par Joël Lebeaume, 1996). La réécriture de ces programmes, entamée en 2002, a abouti à une nouvelle version pour la $6^{e}$ au prin- temps 2004. Le CNP s'est exprimé dans les termes suivants : "Le CNP apprécie la limitation des ambitions dans le domaine de la technologie tertiaire: en effet, il jugeait Celles-Ci démesurées au regard de la maturité des élèves. [...] Quant à l'accent $m$ is sur la conception et la réalisation, il répond à une recommandation souvent répétée, Consistant à mettre les jeunes en contact avec la matière le plus tôt possible. Le CNP préconise une confrontation réelle à la $m$ atière et à l'irréversibilité qui peut exis ter dans son façonnement : c'est un moyen aussi de responsabiliser efficacement les élèves dans leurs initiatives. La technologie avec les sciences expérimentales sont les seuls enseignements qui l'autorisent. Le CNP apprécie donc Cette nouvelle orientation. "Dix ans après ses premières reCom. $m$ andations, le CNP réaffirm e ses préférences.

\section{Le S Condition S né Ce SSaire S pour donner Un aViS}

Le caractère aléatoire des contenus d'enseignement entraîne que n'importe quel enseignant, cherCheur ou inspecteur peut être en désaccord avec les Choix opérés. Il en va bien sûr de même pour le CNP dans sa globalité et pour chacun de ses membres individuellement. La lecture a posteriori d'un projet de programme peut toujours donner lieu à des réactions négatives. Cette situation n'est $p$ as nouvelle, $m$ ais auparavant l'Inspection générale usait implicitem ent de son autorité hiérarchique pour imposer un Changement. Conscient de Cette difficulté, le CNP a souhaité préciser différents éléments de la rédaction des programmes.

Dès l'automne 1991, il est à l'initiative de la Concertation sur la Charte des programmes, qui associe les organisations syndicales, les associations de professeurs spécialistes, les parents d'élèves, les Corps d'inspection et des représentants de l'administration. Le texte définitif est publié au Bulletin officiel de l'Éducation nationale (BOEN) du 20 février 1992. Il donne une définition du programme (8) et explicite quelques principes qui reprennent largement Ceux de la Commission Bourdieu-Gros ; enfin elle indique "Ce qui doit figurer" dans un programme: les objectifs, les connaissances et les Compétences. Ce document, utile pour les groupes d'experts dont souvent les membres n'ont pas de réflexion particulière sur l'objet " programme" (Cf. infra), ne prend en Compte que des Critères de forme.

En 1994, sous la présidence de Luc Ferry, le CNP rédige Les idées direCtrices sur les programmes de 
Collège qui préconise quatre orientations pour en am éliorer la rédaction: hiérarchiser, aléger, harmoniser, carifier. Souvent Cités, Ces quatre principes ne sont en fait que peu utilisés comme Critères de lecture d'un nouveau programme.

L'amélioration la plus importante a été apportée par la décision d'adresser une lettre de c arage, a groupe d'experts Chargé de l'écriture d'un nouveau programme. C'est en 1998 ue Cette pratique est généralisée à l'occasion de la refonte des programmes de lycée. Dans son principe, elle répond à deux exigences : d'abord ne pas laisser le groupe d'experts décider des finalités d'un enseignement, ensuite faciliter l'avis du CNP qui se rapproche ainsi d'un avis de Conformité au cadrage fixé.

Cette lettre est signée par les deux Commanditaires : l'administration par la voix de la Direction de l'enseignement scolaire (DESCO) qui a la responsabilité du calendrier, des conditions de mise en $œ u v r e, d e s$ horaires d'enseignement, de l'articulation avec les examens, etc., et le CNP qui doit s'assurer des Cohérences sur l'ensemble du Cursus, au sein de la discipline mais aussi à chaque niveau d'enseignement ainsi que la prise en Compte des évolutions prévisibles dans la discipline.

Par exemple, la lettre de cadrage adressée au président du groupe d'experts physique-Chimie en 199 , 9indique : "Le programme de seconde [...] doit être reconstruit. Tout objet technique moderne est d'une grande Complexité; tenter d'en analyser sucCessivement les différents aspects donne une fausse idée de la science. [...] il faut en effet y [dans le programme] trouver l'essentiel des notions fondamentales qui vont donner : aux non-sCientifiques, une Culture scientifique minimale dont ils auront besoin, même s'ils n'approfondissent qu'assez peu en première; et aux futurs scientifiques, de très solides bases de départ pour d'importants approfondissements ultérieurs. "Elle précise ensuite, la progression sur les trois années du lyCée. Sur Cette base, Ia mission du groupe d'experts est de trouver les Contenus d'enseignement les mieux adaptés à ces ambitions et d'en assurer une explicitation et une présentation qui soient Compréhensibles par les enseignants.

\section{Une prise de déCiSion Colle ctive}

En dehors de quelques cas, sa Composition pluridisciplinaire a permis au CNP d'émettre des avis utiles et susceptibles d'améliorer la qualité de l'enseignement. La validité d'un programme passe en effet par la possibilité que donne le texte d'être interprété CorreCtement par les enseignants. Il n'est donc pas néCessaire d'être spécialiste de la discipline pour s'interroger sur un programme donné. Dans un passé récent, les avis du CNP ont donc Consisté en général à anticiper les Conséquences des Choix opérés plutôt que de d'évaluer la qualité des Contenus selon une échelle de valeurs qui demeure très incertaine.

L'avis favorable rendu le 7 juin 2005 sur le programme d'histoire-géographie de la série sciences et technologies de gestion (STG) s'inscrit dans Cette logique en précisant : le nouveau programme "représente un progrès réel par rapport au programme en vigueur : l'effort d'allègement est apparent et la possibilité de Choix laissé aux enseignants est appréciable dans une série où les attentes et les intérêts des jeunes peuvent être très divers d'une classe à l'autre". Il en est de même pour l'avis négatif (rendu le 7 juin 2005) sur le programme de $m$ athématiques de la classe terminale de la même série STG : "Le CNP n'est pas d'accord avec ce programme d'une part, parce qu'il ne Comporte pas de lien explicite avec les autres disciplines et avec les objectifs spécifiques de Cette série et d'autre part parce qu'il risque d'encourager une présentation axiom atique des $m$ athématiques à des élèves qui auraient plus besoin de les manipuler que de les théoriser!" Enfin, on peut encore Citer l'avis du CNP pour le programme de philosophie pour les séries technologiques (avis rendu le 7 juin 2005): "Le CNP s'interroge très sérieusement sur les Conséquences que ce projet risque d'avoir dans l'avenir : son imprécision délibérée donne des armes à ceux qui s'accommoderaient volontiers d'une suppression de Cet enseignement! Le CNP ne souhaite pas être le Complice d'une telle dérive".

Dans ces cas, les avis du CNP ne portent pas sur les Choix scientifiques en tant que tels, $m$ ais bien sur leurs Conséquences en termes d'enseignement.

\section{Un e jurisp rud e n Ce discutable}

La proximité du CNP avec le pouvoir politique a parfois eu Comme Conséquence une Certaine forme de transfert de Compétence et de responsabilité : longtemps, un avis négatif du CNP a conduit au retrait du projet de programme et à l'obligation faite au groupe d'experts de le retravailler. L'avis du CNP prévalait sur les choix experts du groupe et parfois sur Ceux des enseignants interrogés dans le cadre des Consultations nationales : alors que la décision de mettre en œuvre un nouveau programme relève 
réglementairement $d u$ ministre, Cette habitude $a m$ is progressivement le CNP en position de Censeur préalable! C'est ainsi que pendant plusieurs années, seuls les programmes ayant reçu un avis positif de la part du CNP ont été soumis au Conseil supérieur de l'éducation et quels que soient les votes de Celui$\mathrm{Ci}$, le $\mathrm{m}$ inistre a déCidé de les faire appliquer en s'appuyant sur l'aval du CNP. Dans Cette procédure, on a fait jouer au CNP une fonction qui ne relève $p$ as de sa Compétence telle qu'elle est fixée dans le décret de Création. Expertiser les Conséquences possibles et instruire l'analyse d'un nouveau programme ne peuvent en aucun cas se substituer à la décision politique qui doit prendre en compte d'autres paramètres telles que l'urgence ou l'opportunité : en attirant l'attention sur les risques encourus, le CNP joue un rôle d'alerte qui peut permettre de prendre les mesures nécessaires pour éviter des Conséquences indésirables. C'est ainsi par exemple qu'en décembre 2003, pour l'ensemble des disciplines technologiques de la série sciences et technologies tertiaires (STT), renom mée à cette occasion STG, le CNP attirait l'attention de la façon suivante : "Le Conseil note que les modifications proposées (Changement d'appellation de la série et des différentes spécialités, nouveaux enseignements, nouveaux programmes) ne sont pas simplement une remise à jour des Contenus liée à l'évolution des entreprises, et qu'elles éloignent Cette formation de l'objectif de pré-professionnalisation spécifique à la voie technologique. Deux Conséquences induites par Ces évolutions ne sont pas suffisamment mesurées et analy. sées : la modification de l'équilibre des filières du second CyCle; la Concurrence entre les différents bacheliers à l'entrée des filières sélectives (STS, IUT) ». Le ministre a préféré, en connaissance de cause, faire appliquer ces nouveaux programmes: la répartition des responsabilités a été en l'occurrence pleinement respectée. Il est toutefois dom mage de Constater qu'en dépit des alertes formulées par le CNP, les dérives n'aient pas été maitrisées : par exemple, dans l'académ ie de Créteil, à la rentrée 2005 , les orientations en classe de première STG accusaient une baisse de $10 \%$ par rapport à Celles en classe de première STT l'année précédente sans que l'on Constate un report vers d'autres séries!

La "jurisprudence " évoquée Ci-dessus n'a pas servi le CNP dans la mesure où des pratiques où seule, la position institutionnelle donne raison sans avoir à se justifier, ne peuvent être bien acceptées mais surtout cela a remis en cause le pouvoir exéCutif qui s'est trouvé dessaisi d'une part de ses prérogatives.

\section{DES RESPONSABILITÉS MAL DÉFINIES}

\section{Le S avis con suitatifs}

Les programmes scolaires sont en France des arrêtés signés par le ministre Chargé de l'Éducation $n$ ationale. Ils sont applicables dans tous les établis. sements publics et privés sous Contrat (lesquels scolarisent plus de $99 \%$ des élèves). La responsabilité de faire appliquer un nouveau programme relève donc du ministre. Pour prendre Cette décision, il dispose du projet et des avis Consultatifs d'une part du Conseil supérieur de l'éducation (CSE) et d'autre part du CNP (Cf. supra).

Le Conseil supérieur de l'éducation est Composé de 97 membres représentant les différents personnels de l'éducation nationale (48 membres), les collectivités territoriales, les associations périscolaires et les grands intérêts Culturels, sociaux et économiques (16 membres), les parents d'élèves et les élèves (19 membres), les organisations syndicales (14 membres). Il est Chargé de donner au ministre un avis sur toutes les questions touchant à l'École. L'avis du CSE se traduit par un vote à main levée : pour, contre, abstention, refus de vote. Les débats qui sont menés sur les programmes dépassent souvent les Contenus d'enseignement. Les syndicats enseignants expriment à Cette occasion leurs inquié. tudes ou leurs désaccords à propos des conditions d'enseignement (horaires, dédoublement, etc.), des Conséquences sur les épreuves d'examen, des besoins de formation des enseignants, etc. Des raisons très variées peuvent donc expliquer un vote négatif du CSE. Globalement, il est assez rare qu'un vote négatif du CSE entraîne le retrait d'un projet de program me.

\section{Le S Con suitation $\mathbf{S}$ nation ale $\mathbf{S}$}

Le projet de programme remis au ministre est le résultat du travail du groupe d'experts qui a souvent multiplié les Contacts : il a reçu l'avis provisoire du CNP encore informel à ce stade, il a rencontré les organisations syndicales et les associations de professeurs de la discipline, mais surtout il dispose des remontées de la Consultation nationale.

Depuis 1994, tous les programmes sont $m$ is en Consultation auprès des enseignants Concernés. À l'origine, C'est pour régler un différend entre universitaires sur les programmes d'histoire et géographie de lycée que la première Consultation nationale a été lancée. Elle a été $m$ al accueillie $p$ ar les organisations 
syndic ke et par l'association des professeurs d'his toire et géographie (APHG) qui ont Contesté ce recours à la démocratie directe.

Dans une première période, les projets $m$ is en Consultation ont été diffusés en nombre dans les établissements ConCernés (écoles, Collèges, lyCées, lyCées professionnels) aveC un questionnaire ouvert. Depuis, les textes sont $m$ is en ligne sur le site internet de la Direction de l'enseignement scolaire ( http://ww w.edusCol.fr).

Pour les programmes de Collège, entre 1995 et 1998, la note d’accompagnement suggérait que les réponses soient rédigées Collectivement au Cours d'une demi-journée banalisée. Les réponses devaient être ensuite adressées dans les rectorats où les inspecteurs territoriaux étaient chargés d'en faire des synthèses au niveau de chaque académie, transmises ensuite à Ia DESCO. Le groupe d'experts disposait ainsi des synthèses académiques et d'une synthèse nationale rédigée par la DESCO.

Dès les premières expériences, les professeurs ont montré un très grand intérêt pour ces consultations même si, parfois, les réponses étaient fortement inspirées par des positions syndicales. Pour le Collège, plus de $80 \%$ des établissements ont répondu régulièrement entre 1995 et 1998. Les professeurs, en tant que "cadres de l'éducation", ont accepté de Contribuer à la prise de décision. Ils ont même eu tendance à outrepasser la demande en s'exprimant sur le Choix des Contenus alors qu'ils étaient interrogés sur leur faisabilité. Cette dérive pose question: appartient-il aux enseignants de Choisir les Contenus d'enseignement? Ont-ils les Compétences pour Cela? Les groupes d'experts ont souvent refusé de leur reconnaître Cette légitimité en tenant compte assez marginalement des remontées. Les Conditions d'exploitation des réponses n'a sans doute pas permis de mettre à la disposition des groupes d'experts, des renseignements vraiment utiles, sauf exceptionnellement. Les synthèses académiques, rédigées en termes choisis et Courtois, ont en effet gommé toutes les aigreurs présentes dans les documents initiaux et elles ont eu tendance à laisser de Côté les suggestions minoritaires: elles ont proposé un discours moyen représentant mal les aspirations particulières du terrain. Ce phénomène a été renforcé dans la synthèse nationale, sorte de "moyenne des moyennes académiques"!

Finalement, les Consultations nationales ont donc peu d'effet sur le détail des programmes mais elles mettent assez bien en évidence les grandes ten- dances et sont à cet égard un élément important de la procédure; elles ont également souvent permis aux groupes d'experts de repérer les points du programme sur lesquels les enseignants s'interrogeaient et sur lesquels les documents d'accompagnement devraient donc apporter des précisions. Elles se sont révélées très utiles pour sensibiliser les professeurs aux nouveaux programmes, et pourdonner aux inspecteurs territoriaux une carte du corps enseignant de leur académie.

\section{L'auto nom ie "irre Sp on sable " de $S$ g ro Upe $S$ d' eXp e rts}

Les programmes scolaires sont des textes très différents dans une discipline particulière selon les formations et les niveaux d'enseignement, et plus encore entre les matières.

Par exemple, deux program mes de m athém atiques ont été présentés au CSE du 7 juillet 2005 . Celui de terminale STG comporte quatre parties: objectifs généraux pour la série STG; m athématiques et usage de l'informatique en première et terminale STG; organisation de l'enseignement et du travail des élèves; le Contenu du programme de terminale STG. Parallèlement, Celui de l'enseignement de spéCialité de terminale littéraire est Construit selon le plan suivant : Introduction (les Contenus disciplinaires, deux domaines transversaux, organisation $d u$ travail des élèves et TICE) ; arithmétique ; analyse ; statistique et probabilités; géométrie ; $\operatorname{argumen-}$ tation mathém atique (analyse du raisonnement, activités algorithmiques).

La Comparaison de Ces deux programmes montre à l'évidence que la composition du groupe d'experts et, en particulier le choix de son président, influe sur le plan retenu. Alors que de nombreux professeurs de lyCées d'enseignement général et technologique seront amenés à enseigner ces deux programmes en parallèle, on ne s'est pas préoccupé des différences de présentation et de leurs conséquences sur la lecture que les enseignants peuvent en faire. En fait, on a Iaissé le soin à chaque groupe d'experts de se forger son propre point de vue sur Ce que doit être un programme de mathématiques de lycée ( 9 )Or, ni les présidents, ni les membres des groupes ne sont recrutés pour Cela : leur expertise porte sur leurs disciplines et parfois sur la didactique de Celles-Ci, m ais pas sur l' habillage qu'il faut donner à un programme pour qu'il soit Correctement mis en œuvre par les professeurs: C'est un autre «métier»! 
Cette situation est le résultat de l'autonomie laissée aux groupes d'experts qui a été Considérée Comme un des aspects importants de la nouvelle procédure $m$ ise en place au début des années $19 \boxplus$. Elle aurait pu fonctionner efficacement si les groupes avaient été responsables de leurs choix devant leurs Com m anditaires (DESCO, CNP), les enseignants, les Corps d'inspection, le $m$ inistre $m$ ême... Or, ils ne rendent de Comptes à personne, se réfugiant derrière leur expertise dis Ciplinaire à la moindre Contestation ; m ême vis-à-vis $d u$ ministre, après que le programme a été discuté au CSE, ils se présentent involontairement en position de force en ne lui laissant que la possibilité du rejet global, souvent impossible à envisager pour des questions de calendrier.

C'est une sorte d'abus de pouvoir involontaire auquel se livrent régulièrement les groupes d'experts. Invités à définir des Contenus d'enseignement, ils outrepassent très vite leur $m$ ission initiale : ils prennent ainsi la place du responsable politique, en fixant les finalités de l'enseignement, Comme par exemple dans le programme de philosophie des séries technologiques (10): "L'enseignement de la philosophie en classes terminales a pour objectif de favoriser l'accès de chaque élève à l'exercice réfléChi du jugement et de lui offrir une Culture philosophique initiale "; ils se substituent aux responsables administratifs et pédagogiques, en affirmant par exemple, dans l'introduction du programme de $m$ athématiques de terminale STG que "Chaque professeur garde toute liberté pour l'organisation de son enseignement, dans le respect du programme...". Les groupes d'experts sont donc amenés à s'exprimer sur des aspects généraux de l'enseignement dont ils n'assumeront ni le suivi, ni l'évaluation et encore moins les éventuelles conséquences.

Cette sorte d'impunité est encore plus flagrante pour les Contenus eux-mêmes. Plus sûrs d'eux que sur les questions générales, ils acceptent $m$ al qu'on les interroge surleurs choix et font souvent des programmes qui sont le reflet de leurs préférences personnelles. C'est ainsi que l'on trouve de l'arithmétique et une référence à la fenêtre de Dürer dans le programme de mathém atiques de terminale littéraire, que l'immunologie prend tout d'un coup une place importante dans les programmes de sciences de la vie et de la terre (SVT) du Collège, que les nouveaux programmes de technologie $d u$ Collège se tournent résolument vers les sciences appliquées... sans que l'on ait étudié les Conséquences de Ces choix sur la formation générale des élèves !

\section{UN SY STÈME SOUS PRESSION}

\section{La décision de Changem ent}

L'intervention des responsables politiques est donc très limitée sur l'évolution régulière des enseignements. Elle se situe principalement dans la décision de changer un programme: pour Cela, le $m$ inistre ne dispose pas de tous les éléments nécessaires. Les groupes de l'inspection générale à travers leurs thèmes de travail annuel en ont quelquesuns; les inspecteurs territoriaux en ont aussi à travers les inspections individuelles; Ia DEP étudie régulièrement les acquis des élèves; Certains services académiques travaillent sur les notes obtenues aux examens; les organisations syndicales interrogent périodiquement leurs adhérents; les associations de professeurs sont très attentives à Ces questions, etc. Il existe donc une multitude d'informations éparses sur l'état d'une discipline et d'un enseignement.

Toutes les informations disponibles devraient être regroupées pour Constituer une sorte de "tableau de bord des programmes ". D' ores et déjà, le ministre pourrait ainsi disposer de l'expertise scientifique indispensable, même si elle ne peut se substituer à la décision politique qui tient Compte d'autres facteurs.

En Complément, des pistes nouvelles devraient être explorées : le suivi des programmes, leur évaluation et peut-être leur expérimentation, Comme on le fait par exemple avant de lancer un médicament sur le m arché, et plus largement l'efficacité des programmes à moyen terme, les effets de l'organisation disciplinaire actuelle (11)...

\section{Le S de m ande $\mathbf{S}$ de la So Cié té}

À Côté de Ce quotidien qui fonde la quasi-totalité de l'enseignement, les dernières années ont vu se multiplier les demandes sociales ou politiques pour que l'école prenne en Charge de nouveaux apprentissages.

On retrouve cela, pour l'éducation sexuelle. Le ministère de l'Éducation nationale a publié la Circulaire "L'éducation à la sexualité dans les écoles, les Collèges et les lyCées" (BOEN, $n^{\circ} 9,27$ février 2003). L'orientation politique de Ce texte est claire dès son introduction: "L'évolution des mentalités [...] a conduit les pouvoirs publics à développer l'éducation à la sexualité en milieu scolaire comme une Composante essentielle de la Construction de la personne et de l'éducation du Citoyen... [...] Cette 
démarche est d'autant plus importante qu'elle est à la fois Constitutive d'une politique nationale de prévention et de réduction des risques..."

Il en est de même pour la sécurité routière. Un enc at publié au BOEN ( $n^{\circ} 40,31$ août 2002 ) est intitulé "Mise en œuvre d'une attestation de première éducation à la route dans les écoles maternelle et élémentaire". Dans son préambule, Cette CirCulaire rappelle que "en 2001, 120000 accidents Corporels ont tué près de 8000 personnes sur les routes de France. La population jeune est particulièrement touchée par l'insécurité routière...". C'est pour répondre à un problème de société que l'École est appelée à intervenir.

Le passage à l'euro le $1^{\text {er }}$ janvier 2002 avait susCité différentes initiatives de la $p$ art $d u$ ministère $d e$ l'Éducation nationale : un BOEN hors série (5 juin 1998) et la Circulaire pédagogique "Préparation au passage à l'euro" (BOEN, $n^{\circ} 34,20$ septembre 2001). Ce texte donne des instructions pédagogiques suivantes : "Le sens de la monnaie et son utilisation font partie des objectifs d'apprentissage de l'école [...] En toute occasion, le sens du passage du franc à l'euro sera rappelé aux élèves. Un lien sera établi avec la construction européenne et son histoire, notamment les traités majeurs instituant l'Union européenne. Une présentation succincte des institutions européennes, et notamment de la Banque centrale européenne, pourra être réalisée. [...] La semaine du 22 au 27 octobre sera particulièrement Consacrée aux opérations liées à Cette ultime préparation. Elle doit Concerner tous les niveaux d'enseignement, de l'école maternelle jusqu'au lycée, tous les publics accueillis en formation dans les établissements et toutes les voies de formation, générale, technologique et professionnelle, ainsi que les personnels de l'éducation nationale, enseignants et non-enseignants. "Dans ce cas, on est entre la dem ande sociale et la décision de responsables politiques dont beaucoup pensaient à l'époque que C'était $p$ ar les enfants que l'on parviendrait à familiariser les adultes à l'euro.

\section{Le $S$ in te rVention S politiq Ue $S$}

Certains enseignements peuvent aussi être introduits ou modifiés à la demande explicite des responsables politiques. Pour l'Éducation à l'environnement pour un développement durable (EEDD), le Président de la République lui-même est intervenu pour demander que cet enseignement soit assuré à tous les jeunes de la maternelle au baccalauréat. La
Circulaire de juillet 2004 BOEN, $n^{\circ} 28,15$ yillet 2004 ) indiqe que la présente Circulaire " s'ins Crit dans la stratégie nationale du développement durable, adoptée par le gouvernement en juin 2003 ". Selon "le souhait du Président de la République, la charte de l'environnement [...] implique la responsabilité de tous. "ICi, I'implication des autorités politiques est explicite alors que les scientifiques sont loin d'être unanimes pour définir les Concepts liés à l'environnement et au développement durable. La tradition française veut qu'un Contenu d'enseignement soit d'abord assuré sur le plan scientifique, en l'occurrence ce n'est pas le cas puisque la déCision politique a prévalu.

Il y a également les injonctions, pas toujours suivies d'effets mais pour autant réelles, émanant d'autres instances. Par exemple, la loi du 23 février 2005 «portant reconnaissance de la nation et la contribution nationale en faveur des français rapatriés" indique dans son article 4 que "les programmes scolaires reconnaissent en particulier le rôle positif de la présence française outre-mer, notamment en Afrique du Nord, et accordent à l'histoire et aux sacrifices des combattants de l'armée française issus de ces territoires la place ém inente à laquelle ils ont droit". Cette loi fixe donc un Contenu d'enseignement ignorant toutes les règles habituelles, notamment qu'il revient au ministre de l'éducation nationale de définir les programmes. Elle a par ailleurs suscité de nombreuses réactions hostiles de la part des universitaires parce qu'elle "impose une histoire officielle, Contraire à la neutralité scolaire et au respect de la liberté de pensée qui sont au Cœur de la laïcité " (12). De l'avis des scientifiques, le pouvoir législatif a manifestement outrepassé ses droits.

L'enseignement de l'Europe fait régulièrement l'objet d'interrogations de la part de parlementaires. Dans le cadre la préparation de la journée de l'Europe, fixée au 9 m ai 2005, la Direction de l'enseignement scolaire a publié une circulaire où l'on peut lire: "Par ses dimensions Culturelle, économique, sociale, universitaire, l'Europe fait aujourd'hui partie de notre vie quotidienne. Les enseignements scolaires, et notamment, mais sans exclusive, Ceux d'histoire, de géographie et d'éducation Civique, juridique et sociale, sont le cadre privilégié de l'information des élèves sur les grandes questions européennes. Les programmes offrent de nombreuses occasions d'aborder divers aspects de l'Europe et de la Citoyenneté européenne. " Dans une période où l'on débattait beauCoup sur l'Europe (13), les enseignants sont rappelés à leurs devoirs. 
L'école est donc de plus en plus sollicitée pour prendre en Charge les préoc opations sociales ou politiques du moment. En répondant à Ces demandes, on fait le Choix de faire suivre par l'École les évolutions de la société, renonçant à les influencer et à les maîtriser. On reporte sur les équipes éducatives et particulièrement sur les enseignants, la responsabilité des nouveaux apprentissages pour lesquels la société, C'est-à-dire souvent les parents, s'est révélée impuissante. Enfin, on introduit ainsi dans les établissements scolaires des Contenus d'enseignement qui n'ont pas d'assises scientifiques : ils ne se réfèrent ni à des savoirs universitaires, ni à des pratiques sociales de référence Codifiées. En l'occurrence, la déCision politique estime pouvoir se passer de l'expertise sCientifique.

\section{La ViSion à long te $\mathrm{rm}$ e}

À Côté de l'adaptation régulière des programmes habituels et des demandes d' "éducation à " qui se règlent au coup par Coup, les Contenus de l'enseignement scolaire nécessitent des mutations plus profondes qui s'inscrivent dans le long terme. Les programmes scolaires sont de plus en plus longs, de plus en plus précis. Les listes de Connaissances sont toujours aussi fournies, les Compétences sont de plus en plus nombreuses et exigeantes... Les professeurs ont de plus en plus de mal à les enseigner, les élèves à les apprendre. La multiplication des sujets supplémentaires à étudier, alliée à ces programmes toujours plus foisonnants, transforme progressivement l'enseignement scolaire en un patchwork dénué de Cohérence et de sens. Depuis près de soixante ans - le Plan Langevin-Wallon (1947) -, revient de façon récurrente la néCessité de reCentrer plutôt que d'éparpiller, d'apprendre à apprendre plutôt qu'apprendre à savoir, apprendre à trouver plutôt que connaître...

Dans Ce contexte général, l'idée de la Culture Commune, du socle Commun ou du socle fondamental est restée longtemps une utopie. Déjà présente dans le rapport Langevin-Wallon en 1947, l'idée est devenue une nécessité avec la $m$ ise en place du Collège unique... en 1975 ! II aura fallu attendre de nouveau trente ans et d'innombrables rapports (14) pour que le principe $d u$ socle soit inscrit dans la loi dans les termes suivants (Loi d'orientation et de programme, 23 avril 2005, art. 9) : "La scolarité obligatoire doit au moins garantir à chaque élève, les moyens nécessaires à l'acquisition d'un socle Commun Constitué d'un ensemble de Connaissances et de Compétences qu'il est indispensable de maîtriser pour accomplir avec succès sa scolarité, poursuivre sa formation, construire son avenir personnel et professionnel et réussir sa vie en société. Ce socle comprend : Ia m â̂trise de la langue française ; Ia maîtrise des principaux élém ents de mathématiques; une Culture humaniste et scientifique permettant le libre exercice de la Citoyenneté ; Ia pratique d'au moins une langue vivante étrangère ; la m aîtrise des techniques usuelles de l'information et de la communication".

Pour la première fois depuis la fin de la seconde guerre mondiale, le Parlement a défini un Contenu d'enseignement en indiquant ce que le socle doit Comprendre. Le rejet de l'idée même du socle par une grande part du corps enseignant - sans doute une majorité - est lié à l'absence de précision sur les objectifs visés. Certains soupçonnent le ministère de vouloir officialiser l'École à deux vitesses: l'école du socle et l'école des milieux favorisés; d'autres y voient les premiers signes d'une remise en cause de Certains enseignements : Ceux qui, Comme I'EPS, les arts ou la technologie, ne sont pas Cités explicitement dans Ia loi; d'autres encore y voient un outil de pilotage du système : une part des moyens d'enseignement pourrait être attribuée en fonction des résultats obtenus au socle...

Ces préoccupations sont justifiées, m ais elles ne portent pas sur la question essentielle de la forme et du Contenu du socle. Ce n'est pas un programme puisqu'il n'est ni annuel, ni disciplinaire. C'est plus qu'une Circulaire donnant un simple cadrage puisqu'il est dans la loi. Alors, différentes hypothèses peuvent être faites : ce peut être un objet d'étude en tant que tel, il faudrait s'assurer alors de la validité du Choix de ses Contenus possibles surle plan épis. témologique; Ce peut être aussi une Clé de lecture des programmes pour les professeurs et les parents d'élèves, il n'est pas sûr que son utilisation ne néCessite pas d'adapter les programmes actuels ; Ce peut être encore un outil de pilotage, mais alors il devra être accompagné de protocoles d'évaluation dont l'utilisation serait obligatoire...

En plus de ces questions générales, la rédaction du socle Commun de Connaissances et de Compétences nécessite une approche globale de la formation, Ce qui est une nouveauté ; elle impose aussi de hiérarchiser les contenus dans chaque discipline et entre les disciplines pour distinguer les points de passage obligés qui permettent aux élèves de poursuivre avec profit leur parcours scolaire...

Les groupes d'experts qui jusqu'à présent ont travaillé sur les programmes ne sont pas armés pour répondre à toutes ces exigences. La rédaction du socle peut donc être l'occasion de revoir la proCédure de réflexion sur les Contenus d'enseignement. 


\section{CONCLUSION}

L'enseignement scolaire s'est longtemps développé sur un petit nombre de disciplines aux références universitaires incontestées. À l'époque, les responsables politiques en avaient délégué la traduction program matique à la hiérarchie pédagogique $d u$ ministère. Quand des références non universitaires ont Commencé à être prises en Compte dans les enseignements et qu'il a fallu Choisir lesquelles retenir, les responsables politiques ont été am enés à jouer un rôle plus important. Au fur et à mesure que la place des politiques se renforçait dans la définition des Contenus scolaires, celle de la science se réduisait, $c o m m e$ si science et politiques ne pou- vaient se retrouver sur les programmes d'enseignement. La rédaction d' "un bon socle commun" nécessitera un rapprochement : un socle scientifique sans choix politique ne sera qu'un "machin " supplémentaire dans le système, et un socle aux objectifs clairement définis ne servira à rien s'il n'est $p$ as Construit sur des b aes scientifiques solides.

Dom inique Raulin dominique.raulin@ac-orleans-tours.fr Directeur du Centre régional de documentation pédagogique de l'académie d'Orléans-Tours Ancien secrétaire général du Conseil national des programmes (2002-2005)

\section{NOTES}

(1) En $2004,52 \%$ des élèves de term inales ont passé un baccalau réat général, $30 \%$ un baccalauréat technologique et $18 \%$ un baccalauréat professionnel.

(2) 249000 candidats se sont présentés au baccalauréat en 1970 et 623000 en 2004

(3) Créées en 1948 en même temps que le Certificat d'aptitude professionnelle (CAP) au niveau national.

(4) Extrait de la lettre adressée à $\mathrm{Ph}$. Joutard, Chargé de la réflexion sur les programmes d'histoire et de géographie.

(5) Un principe n'est manifestement pas rentré dans les faits "toute adjonction devant être compensée par des suppres. sions".

(6) Certains membres ne peuvent être classés de façon Certaine dans une discipline précise

(7) Conseil d'État : section des Contentieux, avis du 13 novembre 2000 : "[L'administration] seule est en mesure d'établir, par la production des procès-verbaux des séances du CNP, que les membres de Cet organisme consultatif ont été régulièrement désignés et Convoqués et que les règles de quorum on été respectées".

(8) “Le programme est un texte réglementaire, publié au $B O$ : C'est le texte officiel qui sert de référence nationale pour fonder $\boldsymbol{d}$ an $\boldsymbol{S}$ chaque discipline, à chaque niVeau, le "Contrat d'enseignement ", C'est-à-dire le cadre à l'intérieur duquel l'enseignant ou l'équipe pédagogique font les choix pédagogiques adaptés aux élèves dont ils ont la responsabilité. Il a par ailleurs pour fonction d'établir une clarification entre les différents niveaux du système éducatif et de définir les compétences que les élèves doivent acquérir. " (France, 1992, p. 488)

(9) Il est possible que la forme pour le Collège ou pour le LP doive être différente.

(10) Ce programme a été présenté au CSE du 7 juillet 2005

(11) Par exemple, le positionnement de la géographie, le couplage physique et Chimie, Ia disCipline scolaire "sCiences éConomiques et sociales", la place de la philosophie uniquement enseignée en term inale...

(12) C. Liauzu, G. Meynier, G. Noiriel F. Régent, T. Van Thao, L. Valensi, "Colonisation : non à l'enseignement d'une histoire officielle ", Le Monde, $25 \mathrm{~m}$ ars 2005 .

(13) La France a voté par référendum sur le projet de Constitution européenne, le $29 \mathrm{~m}$ ai 2005 .

(14) Le dernier en date est Celui de la "Commission nationale pour l'avenir de l'école", en 2004.

\section{BIBLIOGRAPHIE}

Bourdieu P. \& Gros F. [dir.] (1989). Principes pour une réflexion sur les Contenus de l'enseignement / Commission présidée par Pierre Bourdieu \& François Gros. [Paris] : [lm primerie nationale], $14 \mathrm{p}$.

Collège de France (1985). Propositions pour l'enseignement de l'avenir / élaborées à la dem ande de Monsieur le Président de la République $p$ ar les professeurs du Collège de France (1985). Paris: Collèg e de France.

Demonque C. [dir.] (1994). Qu'est-Ce qu'un programme d'enseignement? Paris: CNDP ; Hachette éducation.

France : Ministère de l'Éducation nationale (1990). Décret $n^{\circ} 90-179$ di 23 février 19 co instituant le Conseil natio$n$ al des program mes. [NOR : MENL9000312D].

France : Ministère de L'Éducation nationale (2 002 ). "Ch arte des programmes". Bulletin officiel de l'Éducation natio$n$ ale, $\mathrm{n}^{\circ} 8, \mathrm{p} .487-492$ [NOR : MENW9250078X]
ISAMBERT-JAMATI V. (1995). Les savoirs scolaires: enjeux sociaux des Contenus d'enseignement et de leurs réformes. Paris: L'Harm attan. [ $1^{\text {re }}$ éd. Paris : Éd. universitaires, 1990 ]

LeBEAUME J. (1996). "Une discipline à la recherche d'ellemême : trente ans de technologie pour le Collège". Aster: recherches en didactique des sciences expérimentales, $\mathrm{n}^{\circ} 23$, p. 9-41.

Legrand L. (1982). Pour un Collège démocratique. Rapport remis au ministre de l'Education nationale. Paris : La Docum entation française.

Prost A. (1992). Sociétés et politique : une histoire de l'enseignement en France en 194 के nos jours. Paris: Éd. du Seuil.

RAULIN D. (2006). Les programmes scolaires. Paris : Retz.

Robert A. D. (1993). Système éducatif et réformes : de 1944 ànos jours. Paris: Nathan. 